\title{
Precision oncogenomics
}

\author{
Andrea Ferreira-Gonzalez ${ }^{1}$ and Elaine R. Mardis ${ }^{2}$ \\ ${ }^{1}$ Virginia Commonwealth University, Richmond, Virginia 23284, USA; ${ }^{2}$ Nationwide Children's Hospital and \\ the Ohio State University, Columbus, Ohio 43215, USA
}

Decades of research has led to the knowledge that cancer is a genetic disease driven by the accumulation of molecular alterations that negatively impact control of cell division (Vogelstein et al. 2013). This understanding of cancer as a genetic disease has led to a focus on the genomes of cancer cells, looking for the leading cause(s) of the pathological cellular proliferation that ultimately results in cancer. Early work identifying specific driver genomic alterations led to the development of targeted therapies, more effective and less toxic compared to standard chemotherapies. Trastuzumab (FDA-approved in 1998) and imatinib (FDA-approved in 2001) were the first two drugs to illustrate the potential of targeted therapy for the treatment of breast cancer and chronic myeloid leukemia $(\mathrm{CML})$, respectively. These early successes were followed by a multitude of small molecule inhibitors that have FDA approval for the treatment of many types of cancer (Fischer et al. 2003). Today, both imatinib and trastuzumab are cited as "essential medicines" by the World Health Organization, available as a generic and biosimilar, respectively.

Coincident with the emergence of targeted therapy for cancers, the sequencing and assembly of the human genome nearly two decades ago heralded the advent of massively parallel sequencing technologies capable of producing enormous quantities of genomic data. The success of the Human Genome Project enabled the application of next-generation sequencing (NGS) technologies and bioinformatics tools to identify genome-wide alterations. NGS allowed faster and simpler sequencing by using microscopic, spatially separated DNA templates to massively parallelize the capture of data. With such platforms in hand, it became possible to sequence all the coding exons of a genome and even a whole human genome in a short time and at an affordable price. In addition, bioinformatics tools were developed that allowed the processing and analysis of these data with high accuracy and reproducibility. With a Human Genome Reference in hand, it finally became possible to catalog the pathogenic alterations in cancers, to discover frequently altered genes and their putative role in cancer, and to identify "druggable" targets (i.e., genes with mutations known to be responsive to targeted therapies). Furthermore, the development of publicly available databases of cancer genomic data, such as The Cancer Genome Atlas (TCGA), has been instrumental in the discovery of novel cancer-associated genes and advancing the field of translational cancer genomics by cataloging genes and variants from more than 40 common human cancers. Cumulatively, these developments in technology, bioinformatics, therapeutics and databases have given birth to the "genomic era" of cancer research. Of course, the disadvantage of this comprehensive cataloging is that NGS often identifies new variants in genes that we cannot readily interpret in terms of their pathogenicity or likely response to therapy. Coincidentally, this is one of the strongest arguments for a journal like Cold Spring Harbor Molecular Case Studies_namely, the rapid communication of new variants and/or therapeutic responses. This special issue offers several such case reports as examples.

The ability to deeply sequence the genomes of cancers, at rapidly decreasing cost, has provided translational cancer researchers with unprecedented opportunities to gain insights into the biology of cancer initiation, progression, identification of actionable somatic and

Published by Cold Spring Harbor Laboratory Press

doi:10.1101/mcs.a004150 
germline variants, and even the identification of genomic changes conferring acquired resistance to these therapies. Whole-genome and -exome sequencing studies of primary and metastatic tumors in different cancers have revealed intratumoral heterogeneity as a key driver of acquired resistance and clonal evolution (Jamal-Hanjani et al. 2017). Furthermore, NGS technology has allowed us to identify alterations even in scarce biopsy tissues or in simple blood draws (liquid biopsy) for cases in which collecting tissue for molecular testing might be unsafe for the patient (Ignatiadis and Dawson 2014). This special issue contains several such reports of the use of liquid biopsy for patient diagnosis or monitoringevidence that this approach is being used with increasing frequency in the clinical setting.

These advances in cancer genomics have paved the way for a new era of precision medicine in oncology —an era in which a patient's tumor can be characterized extensively for mutations and other molecular abnormalities, and treatment can be based on the identified molecular changes along with the cancer's tissue of origin. It is not a new concept, but the use of NGS, bioinformatics tools, and large-scale cancer variant databases have created an opportunity for significant onward movement of this approach.

In current clinical practice, providers encounter the growing challenge of interpreting variants for each patient, who may have one to 10 somatic mutations involving 50 to 500 genes. Furthermore, the types of mutations that can be detected continue to expand. The BRAF oncogene provides an illustration of this challenge. This oncogene may contain mutations that either lead to kinase activation (Class I and II point mutations), kinase-dead activity (Class III point mutations), kinase domain duplication, or may be involved in a gene fusion. Each of these classes of mutations may be responsive to a RAF inhibitor, MEK inhibitor, or combination therapy depending on the biology it determines. However, sifting through these mutations, biological evidence, and potential matching therapies is a time-consuming and arduous task that lacks harmonization and guidelines. This complex process, called variant prioritization, is aided by large publicly accessible databases like CIViC (Griffith et al. 2017), the GA4GH VICC (Wagner et al. 2018), or OncoKB/cBioPortal (Chakravarty et al. 2017), among others. These resources have been designed as a translational bridge between researchers and clinicians, to lower the barriers of access and make comprehensible the individual genes and their alterations with functional and/or drug therapy responses. However, because the reality is that functional consequences are known for only a small fraction of the many variants identified by NGS, increasingly there are decision-support tools emerging to facilitate the interpretation of cancer genomes in this context. Two of the case reports in this issue highlight how such tools can be utilized in cancer precision medicine.

Each incremental advance in cancer knowledge is ultimately an improvement in patient care. Although the scientific value of NGS-based advancements is undoubtedly critical, the derived clinical benefits are still being discussed. Nevertheless, NGS is driving the field of translational precision cancer medicine at a rapid pace, coupled with increasing evidence from patient-based studies and ensuing therapeutic management strategies. This issue of Cold Spring Harbor Molecular Case Studies provides insights into the current state and clinical practice of precision oncology, the challenges that we currently face, and the enormous opportunities to come. These contributed manuscripts provide a glimpse into our understanding of novel genomic features such as druggable fusions, new analytical approaches, the complexity of data generated, and how to interpret these complex data in the context of patient care.

We are thankful to the many groups and individuals who contributed their work and points of view to this issue, and also to the patients who consented to have their stories told in order to benefit all future patients. We hope that you will find these contributions en-

Competing Interest Statement

The authors have declared no competing interest. lightening regarding the current status of precision oncology and inspirational of what is to come. 


\section{REFERENCES}

Chakravarty D, Gao J, Phillips SM, Kundra R, Zhang H, Wang J, Rudolph JE, Yaeger R, Soumerai T, Nissan MH et al. 2017. OncoKB: a precision oncology knowledge base. JCO Precis Oncol 1: 1-16. doi:10.1200/PO.17 .00011

Fischer OM, Streit S, Hart S, Ullrich A. 2003. Beyond herceptin and gleevec. Curr Opin Chem Biol 7: 490-495. doi:10.1016/S1367-5931(03)00082-6

Griffith M, Spies NC, Krysiak K, McMichael JF, Coffman AC, Danos AM, Ainscough BJ, Ramirez CA, Rieke DT, Kujan $\mathrm{L}$, et al. 2017. CIViC is a community knowledgebase for expert crowdsourcing the clinical interpretation of variants in cancer. Nat Genet 49: 170-174. doi:10.1038/ng.3774

Ignatiadis M, Dawson SJ. 2014. Circulating tumor cells and circulating tumor DNA for precision medicine: dream or reality? Ann Oncol 25: 2304-2313. doi:10.1093/annonc/mdu480

Jamal-Hanjani M, Wilson GA, McGranahan N, Birkbak NJ, Watkins TBK, Veeriah S, Shafi S, Johnson DH, Mitter R, Rosenthal R, et al. 2017. Tracking the evolution of non-small-cell lung cancer. N Engl J Med 376: 21092121. doi:10.1056/NEJMoa1616288

Vogelstein B, Papadopoulos N, Velculescu VE, Zhou S, Diaz LA Jr, Kinzler KW. 2013. Cancer genome landscapes. Science 339: 1546-1558. doi:10.1126/science.1235122

Wagner AH, Walsh B, Mayfield G, Tamborero D, Sonkin D, Krysiak K, Deu-Pons J, Duren R, Gao J, McMurry J, et al. 2018. A harmonized meta-knowledgebase of clinical interpretations of cancer genomic variants. bioRxiv 366856. doi:10.1101/366856 


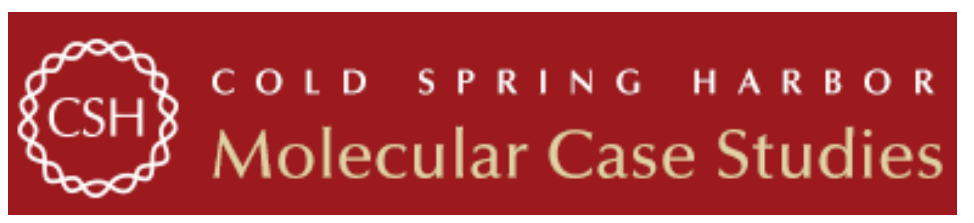

\section{Precision oncogenomics}

Andrea Ferreira-Gonzalez and Elaine R. Mardis

Cold Spring Harb Mol Case Stud 2019, 5: a004150

Access the most recent version at doi: $10.1101 /$ mcs.a004150

References This article cites 7 articles, 2 of which can be accessed free at:

http://molecularcasestudies.cshlp.org/content/5/2/a004150.full.html\#ref-list-1

License This article is distributed under the terms of the Creative Commons Attribution-NonCommercial License, which permits reuse and redistribution, except for commercial purposes, provided that the original author and source are credited.

Email Alerting Receive free email alerts when new articles cite this article - sign up in the box at the Service top right corner of the article or click here. 